\title{
An Operating Environment for Control Systems on Transputer Networks
}

\author{
H.G. Tillema, A.L. Schoute, K.C.J. Wijbrans \\ Mechatronics Research Centre Twente, University of Twente
}

\begin{abstract}
This article describes an operating environment for control systems. This environment contains the basic layers of a distributed operating system. The design of this operating environment is based on the requirements demanded by controllers which can be found in complex control systems. Due to the nature of real-time control systems, special attention has to be payed to an efficient implementation of the basic communication protocol and buffering method. This operating environment has been implemented and performance measurements have been carried out for several different strategies.

The described operating environment which is used for robot control, will be embedded in a larger high level transputer operating system.
\end{abstract}

Keywords: real time operating systems, transputers, robot controllers, parallel processing, performance measurements.

\section{Introduction}

At the Mechatronics Research Centre of the University of Twente, several kinds of robots are designed and investigated such as a two-link flexible robot arm, a high-speed sixlink robot arm and a mobile autonomous robot system. For the fast and accurate control of such systems, advanced control algorithms have to be used which are computational intensive. The controllers used for robot control often operate at sampling frequencies between 250 and $1000 \mathrm{~Hz}$. It will be obvious that these high sample frequencies introduce hard real-time demands.

Generally a control system consists of different components covering multiple (hierarchical) control loops, transformation and protection layers, etc. In order to meet the real-time demands these components have to run in parallel. At our research centre, a choice has been made to use transputers. One of the advantages of using transputers is the ease at which parallel processing facilities are made available, both with respect to hardware and software. With respect to the software, a control system consists of several different control modules. These modules generally will be developed independently, and have to be connected together in a flexible way. Therefore an operating environment has been designed in which the different modules of the system can be embedded separately. The modules encompass parts of the control system that will run in parallel on the transputer network. A single transputer itself is already a fast microprocessor with hardware facilities for parallel processing. This implies that the decision to allocate parallel processes for the same or different nodes is a matter of design and performance trade-off.

The operating environment has been developed for a transputer network of arbitrary size and configuration. The main function of the environment is to provide communication between the modules itself and the outside world. It has been written in OCCAM, the 'native' language of the transputer. A detailed description of it can be found in [TIL89].

\section{General control system requirements}

A control system can in general be depicted as consisting of several interacting control loops. Each control loop consists of a process and a controller. The controller obtains a setpoint and the measurements of the process. By comparing these and applying a suitable algorithm, the process can be controlled in a satisfactory manner. Within one sample time, the communications between the process and the controller and the computations have to be performed as illustrated in figure 1 .

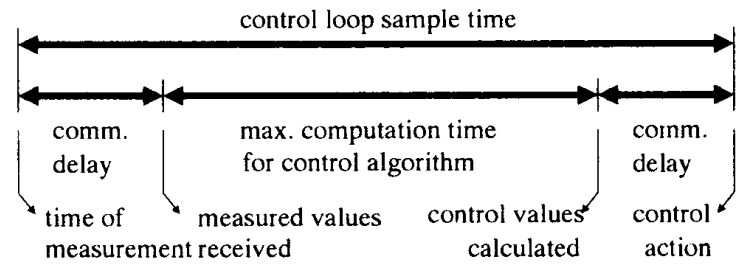

figure 1: a control loop sequence 


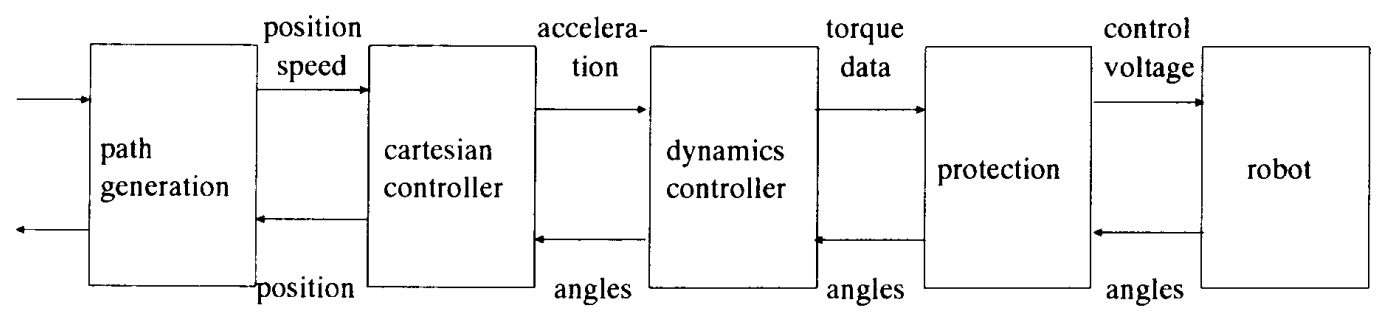

figure 2: a robot control system

Typical for more complex control systems is their hierarchical structure. This structure can be found at almost all levels in the system. An example of a typical robot controller is given in figure 2 .

The different independent parts communicate setpoint data and measurements with one another. Furthermore, in practice some supervisory system will be added, that is monitoring the behaviour of the control modules and that can send commands. In such a system, two different data streams can be noted:

\section{- The control data}

The control data consists of the setpoints and the measurement values that are exchanged between the control modules implementing the control loops. Typical for this data stream is its time synchronous nature. The rate at which the control data will be communicated from one module to another is determined by the sample frequency of the control loop. At each sample interval a setpoint and a set of measurements must be exchanged. Intermediate buffering is time constrained, the control data must arrive at the destination within a sample interval. Buffering between different controller modules could introduce dead time in the system, which influences the behaviour of the control system and may even result in instability. Furthermore, the delay in this data transfer must be kept to a minimum to leave sufficient time for the computation of the control action.

- The command and status messages

The command and status messages have a different nature. These messages are exchanged between some supervisory system and the control modules, and provide information for the change of the mode of the control modules and the monitoring of the status of these modules. These messages arrive asynchronously. In this case intermediate buffering is allowed. The delay in the communication is not critical.

\section{Architecture of the operating environment}

The operating environment provides on each node of the transputer network a framework in which a control module can be embedded dynamically, i.e. at run-time. Such a module is an independent OCCAM program specified by the user. The environment can be divided into three layers as shown in figure 3 .

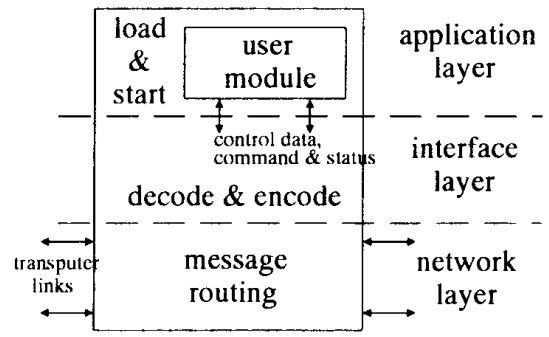

figure 3: the operating environment architecture

The bottom layer -called network layer-contains all the processes necessary for the message routing through the system. This layer is present on each transputer in the system. The second layer in the system - called interface layertakes care of protocol and address conversion and contains some application managing tasks.

The third layer is the application layer. In this layer, the control module can be loaded and started.

\subsection{The addressing scheme}

To perform communication between the modules, some kind of addressing is needed.

The addressing scheme used in the operating environment is an indirect addressing scheme. Within a control module logical destination addresses are used to refer to other control modules. These logical destination addresses are translated to physical destinations (transputer numbers) by the environment. This translation is module independent and is specified separately in a project file. This project file can be seen as a description of the software structure. By changing the project file, the system can be adapted to other hardware configurations without changing the control modules themselves. In this way, a decoupling of the software and hardware structure has been established. This is especially useful if a more complicated controller structure is used instead of 


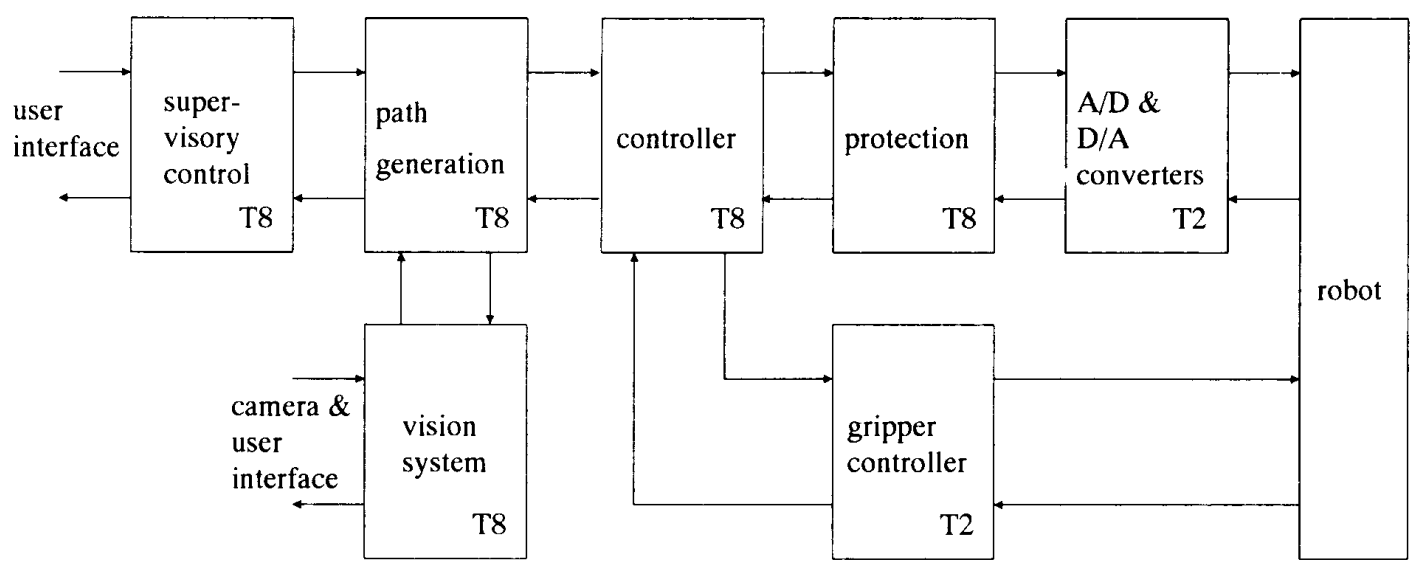

figure 5: an example of a more complex system

a simple pipeline (as in figure 2). An example of such a system is shown in figure 5 .

\subsection{The network layer message formats}

To reach a high performance, the efficiency of the network protocol is very important. In a first implementation of the operating environment, a variable length message format was used to minimize the amount of data which had to be sent between transputers. In this implementation a message consisted of three parts, a header, a size byte and the actual data.

To reduce the number of communications for a message, the header and the data were combined into one part. The speedup reached by this improvement was the reason to change the message format to a fixed size. The size byte in the message still gives the actual size of the data field, the rest are padding bytes.

The different formats are shown in figure 4.

A

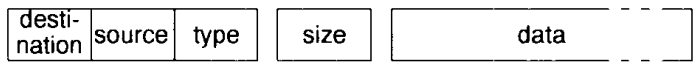

B

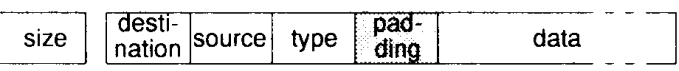

C

\begin{tabular}{|l|l|l|l|l|}
\hline $\begin{array}{l}\text { desti- } \\
\text { nation }\end{array}$ source & type & size & data & $\begin{array}{c}\text { padding } \\
\text { bytes }\end{array}$ \\
\hline
\end{tabular}

figure 4: the different message formats

\subsection{Buffering schemes}

The network and interface layers of the system need some kind of buffering. In order to maximise the performance of the system, three different buffering techniques were investigated. These techniques differ in the way messages are saved in an array and the way the input and output processes communicate with each other. (Due to the OCCAM concept, different input and output processes are needed because it is not possible to check if input is available without waiting for it.)

The first buffer type(I) uses array retyping to avoid shared memory between the input and output process. The array retyping must be performed after each outputted message to meet the OCCAM conventions. Because of the time needed for the array retyping and the starting and ending of the two parallel processes, the performance of this buffer is relatively poor.

The second buffer type(II) uses a shared array for the parallel processes with communications between the processes at each message for administration purposes.

The third buffer type(III) is a so-called sleeping barber buffer[BEN82]. Both processes use a part of a common area for administration purposes. Because both processes run at high priority, mutual exclusion of the processes is guaranteed. As soon as a proces becomes idle, it will deschedule and wait until it is restarted by the other proces.

\section{Real-time Performance}

Besides the control module each node contains a communication part consisting of a number of communication processes. These processes take care of message transfer, protocol handling and buffering. As a result each node runs a mixture of communication and application processes.

Because of the hard real-time control requirements the performanc

of the system has been investigated for five different configurations. These configurations are combinations of the different message formats and buffering schemes. The following performance indicators were chosen (See also [ROE89]): 


\begin{tabular}{|c|c|c|c|}
\hline & buffer I & buffer ll & buffer III \\
\hline protocol A & $\begin{array}{l}\text { throughput }=6.2 \mathrm{kpackets} / \mathrm{s} \\
\text { delay }=274 \mu \mathrm{s} \\
\text { add. delay }=121 \mu \mathrm{s}\end{array}$ & & \\
\hline protocol B & $\begin{array}{l}\text { throughput }=6.2 \mathrm{kpackets} / \mathrm{s} \\
\text { delay }=252 \mu \mathrm{s} \\
\text { add. delay }=112 \mu \mathrm{s}\end{array}$ & $\begin{array}{l}\text { throughput }=6.2 \mathrm{kpackets} / \mathrm{s} \\
\text { delay }=178 \mu \mathrm{s} \\
\text { add. delay }=110 \mu \mathrm{s}\end{array}$ & \\
\hline protocol C & & $\begin{array}{l}\text { throughput }=10.4 \mathrm{kpackets} / \mathrm{s} \\
\text { delay }=143 \mu \mathrm{s} \\
\text { add. delay }=88 \mu \mathrm{s}\end{array}$ & $\begin{array}{l}\text { throughput }=10.4 \text { kpackets } / \mathrm{s} \\
\text { delay }=138 \mu \mathrm{s} \\
\text { add. delay }=81 \mu \mathrm{s}\end{array}$ \\
\hline
\end{tabular}

table 1: measurements for different configurations

- The maximum sample throughput between neighbouring transputers

This maximum sample throughput determines the speed at which control loops can be executed. A fixed packet $\operatorname{size}(76$ bytes; the largest packet size in the current application) was chosen. This is the packet size used for the control data.

- The worst-case delay between neighbouring transputers for a given throughput

This delay determines the amount of time left in the sample interval of the control loop for computations as shown in figure 1 . It has been measured as a function of different throughput values.

- The additional delay for each extra transputer between two communicating transputers

This delay restricts the amount of computational time left for some control modules.
- The processor utilization as a function of the throughput.

The processor utilization determines the amount of computational effort a controller module can perform on a communicating transputer.

In order to satisfy the real-time constraints the following approach is taken:

- The communication is made as efficient as possible. The different buffering techniques and message protocols are compared in terms of the first three performance indicators. For details we refer to [WYB90]. An overview of the results is given in table 1 . Measurements showed that the delay values are almost independent of the sample throughput rate.

- In order to minimize message delays communication processes run at high priority whereas the application processes run at low priority.

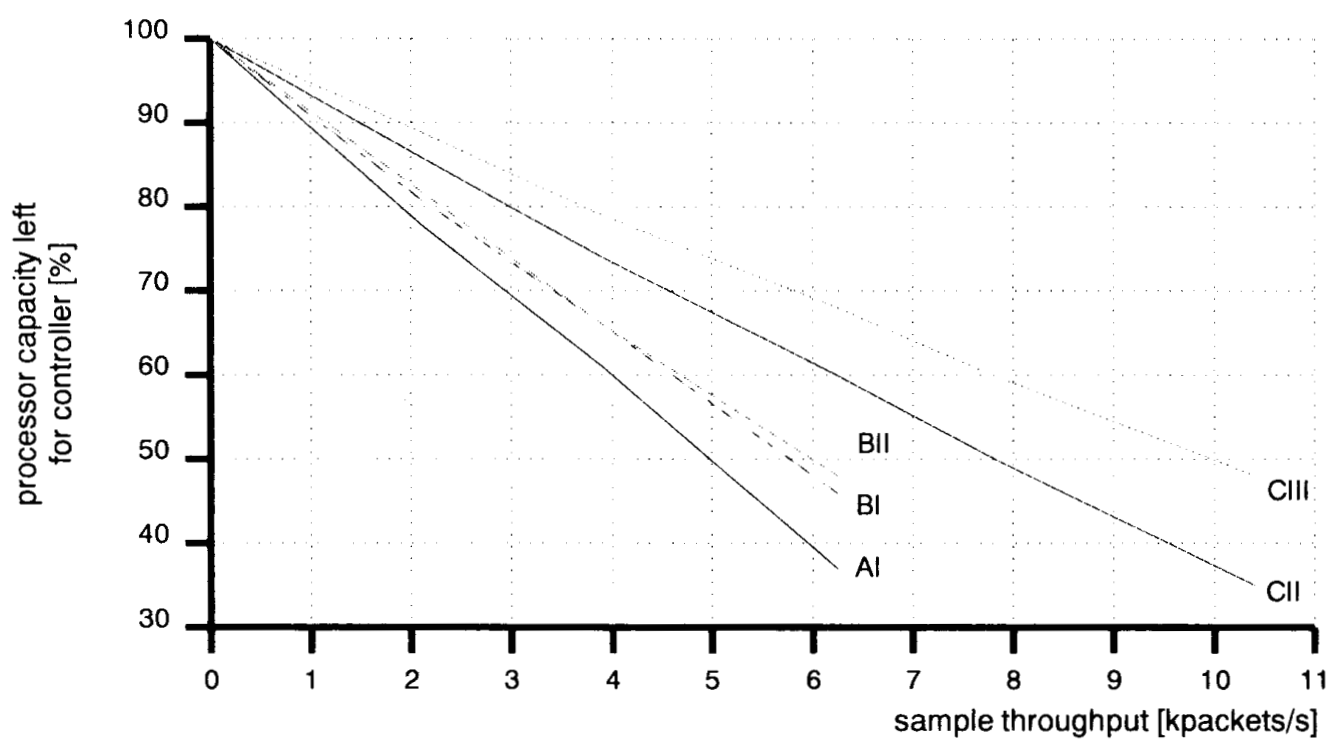

figure 6: utilization versus throughput 
- The processor capacity available for the application processes is guaranteed to a fixed level according to a clear trade-off between sample throughput and processor utilisation. According to the measurements a linear relationship exists between the sample throughput and the resulting communication overhead. This relationship is given in figure 6 for the different cases of message protocols and buffering techniques.

\section{Conclusions and future developments}

The operating environment described in this paper operates well in practice. Due to the small delay, the added flexibility and the small code size it is very well suited for real-time control applications. Currently, the system is used for this purpose at the University of Twente in a configuration which resembles figure 5 strongly.

The goals of combining a high maximal sample throughput with a low delay have been reached for the last implementation. Due to this, the system can be used for the operation of neighbouring controller modules at rates of $1 \mathrm{kHz}$. For an intermediate controller, which has to handle $2 \mathrm{kpacket} / \mathrm{s}$ in both directions, the utilization by the communications network is then still restricted to $10 \%$, leaving a controller performance of $90 \%$.

Because the operating environment is intended specifically for control system applications, worst case performance indicators and measurement methods have been chosen such that the actual performance can be predicted reliably.

From the experiences with the operating environment implementation it can be concluded that it is important to keep the efficiency of communications protocols and buffering strategies in mind. The delay is reduced significantly by choosing a protocol with a minimal number of communications. For small packet sizes this is more important than the actual size of the communication due to the communication set-up times. The buffering strategy chosen can influence the processor utilization of the system very much. An efficient buffering strategy, that performs a minimal number of actions for each message received, appears to be the so-called sleeping barber buffering strategy.
Due to its speed and real-time characteristics the described system offers an ideal environment for the lower levels of robot motion control. At higher levels of motion control (task planning) the real-time requirements are less severe and other requirements become more prominent, such as general file $\mathrm{I} / \mathrm{O}$ facilities and general purpose task scheduling. A (not hard real-time) distributed transputer operating system - named TROS - has been developed for this purpose[OEL90]. The control environment as described here may in future be embedded in TROS and can run on particular transputer subnetworks of the total network configuration. In this respect a two-level hierarchy of operating environments (ranging from general purpose to real-time specific) is created.

The advent of the $\mathrm{H} 1$ transputer will greatly influence the performance of the system. In this case, a part of the functionality of the communication processes will be implemented in hardware. Together with the through-routing chips, this will provide a dramatic increase in the performance of the system.

\section{Literature}

[BEN82] Ben-Ari M., "Principles of Concurrent Programming", Prentice-Hall, 1982.

[OEL90] Oelen W., "The transputer operating system TROS - Reference manual", Memoranda Informatica 90-68, University of Twente, November 1990, ISSN0923-1714.

[ROE89] Roebbers $\mathrm{H}$. and M. Vlot, "A communication processor on the transputer", Proc. 10th Occam User Group Meeting, p.143-151, Enschede, April 1989.

[TIL89] Tillema H.G., "The development and implementation of an operating environment for control systems", M.Sc.thesis, Control Laboratory, Dept. of El.Eng., University of Twente, rep. no. 89R187, 1989.

[WYB90] Wijbrans K.C.J., Tillema H.G., Bakkers A.W.P., Schoute A.L., "An operating environment for control systems", Proc. 13th Occam User Group Meeting, York, September 1990. 